\title{
ST Segment Depression by ECG Finding
}

National Cancer Institute

\section{Source}

National Cancer Institute. ST Segment Depression by ECG Finding. NCI Thesaurus. Code C41330.

An electrocardiographic finding of ST segment depression below the baseline, often described as up sloping, down sloping or horizontal. 